\title{
A clinico-mycological study of onychomycosis at a tertiary care center
}

\author{
Attar Farhana Iqbal', Tendolkar Uma Mahesh'1, Baveja Sujata Mukesh', Dhurat Rachita², \\ Phiske Meghna ${ }^{2}$ \\ Departments of ${ }^{1}$ Microbiology, and ${ }^{2}$ Dermatology \& Venereology, Lokmanya Tilak Municipal Medical College and
} General Hospital, Sion (West), Mumbai, Maharashtra, India

Background: Onychomycosis continues to be worldwide problem constituting a large bulk of cases attending the dermatology outpatient department. Fungal infections of nails have been partly studied, because it has been considered more of a cosmetic problem than a health problem. Although not life threatening, onychomycosis may have significant clinical consequences such as secondary bacterial infection, chronicity, therapeutic difficulties and disfigurement, in addition to serving as reservoir of infection. Aims and Objectives: 1) To find out the prevalence, etiology and clinico-mycological correlation of onychomychosis among clinically suspected cases. 2) To compare the microscopy findings using potassium hydroxide $(\mathrm{KOH})$ preparation with potassium hydroxide- dimethyl sulfoxide (KOH-DMSO/ DMSO) preparation. 3) To compare demographic and clinical data in onychomycosis and fungus negative groups. Material and Methods: The study was conducted from October 2011 to October 2013, in the department of Microbiology of a tertiary care hospital in Mumbai, including 204 patients clinically suspected of onychomycosis. Nail scrapings were collected depending upon site, type of nail involvement and subjected for microscopic examination using $20 \% \mathrm{KOH}$ and $\mathrm{KOH}$ DMSO $(10-40 \%)$, followed by fungal culture. Results: Out of 204 patients, 78 (38.2\%) were diagnosed having onychomycosis, 72 (92.3\%) patients were positive by direct microscopy and $45(57.7 \%)$ by culture. DMSO preparation offered a faster clearing of background but in $\mathrm{KOH}$ preparation fungal morphology was better maintained even after 24 hours. In culture positive cases, isolation of dermatophytes was most common (42.2\%), followed by Candida species $(31.1 \%)$ and nondermatophytes $(26.7 \%)$. Conclusion: This concludes that laboratory diagnosis of onychomycosis is essential as many conditions of nail mimic onychomycosis. Though commonest causative agents of onychomycosis are dermatophytes, number of cases caused by Candida species and nondermatophytesare on the rise.

Key words: Onychomycosis, Nondermatophytes, Dermatophytes, Di-methyl sulfoxide (DMSO), Potassium hydroxide $(\mathrm{KOH})$

\section{INTRODUCTION}

Diseases of nails can be inherited or acquired. They may be a part of local or systemic pathology. It is essential to differentiate onychomycosis from other nail disorders as certain skin conditions such as psoriasis, lichen planus, onychogryphosis and nail trauma can mimic onychomycosis. The term onychomycosis (OM) is presently used to describe nail infection caused by dermatophytes, yeast and nondermatophyticmoulds. Until about two decades back, onychomycosis was a poorly discussed topic. ${ }^{1}$ Extensive laboratory investigations are needed to differentiate accurately between fungal infections and other nail conditions. It requires long term antifungal treatment which is also expensive, so the correct diagnosis before commencement of therapy is needed.

Fungal infections of nails have not been extensively studied partly because it has been considered more of a cosmetic problem than a health problem. ${ }^{2}$ Although 
not life threatening, onychomycosis may have significant clinical consequences. Onychomycosis is the most common of all nail disorders, accounting for up to $50 \%$ of all onychopathies and about $30 \%$ of all cutaneous fungal infections.

Systematic studies on OM are few and controlled studies are rare even in recent times in our country. Our study includes a comparative observation of patients with OM and the fungus negative group. Clearly defined terms of OM that were followed, periodic observation of direct microscopic preparations using Potassium hydroxide $(\mathrm{KOH})$ and potassium hydroxide- dimethyl sulfoxide (KOH-DMSO) and photographic documentation of yeasts in nails are some features which are the highlights of the present study.

\section{Aims and objectives}

1) To find out the prevalence of onychomychosis among clinically suspected cases.

2) To compare the microscopic findings using potassium hydroxide $(\mathrm{KOH})$ preparation with potassium hydroxide- dimethyl sulfoxide (KOH-DMSO/DMSO) preparation.

3) To find out the prevalence of dermatophytic, nondermatophytic and candidialonychomychosis.

4) To correlate clinical and microbiological findings.

5) To compare demographic and clinical data in onychomycosis and fungus negative groups.

\section{MATERIALS AND METHODS}

The study was conducted in the department of Microbiology of a tertiary care hospital in Mumbai over the period of two years from October 2011 to October 2013. A total of 204 patients with suspected onychomycosis on clinical examination were included in study. Informed consent and a detailed clinical history were recorded. Cutaneous examination was carried out with detailed examination of the nail unit. Patients who were suspected clinically of having onychomycosis were included in this study. Patients, not been clinically suspected of onychomycosis and also patients on antifungal treatment were not included.

\section{Specimen collection}

After proper washing and disinfection using spirit, sample collection was done by scraping of nail, depending upon the site from maximally affected area.

Processing of specimen

Direct microscopy was done after adding the nail specimen to the:
1) $20 \%$ Potassium hydroxide $(\mathrm{KOH})$ solution and

2) $\mathrm{KOH}-\mathrm{DMSO}$, referred to as DMSO: $40 \mathrm{ml}$ of DMSO mixed with $60 \mathrm{ml}$ water, to this $10 \mathrm{gm}$ of $\mathrm{KOH}$ was added. ${ }^{3}$

Specimens were observed under microscope after 10 minutes, one hour and 24 hours in moist condition.

All the nail specimens were cultured on

1) Sabouraud dextrose agar (SDA), with chloramphenicol and gentamicin and the tubes were incubated at $25^{\circ} \mathrm{C}$.

2) Sabouraud dextrose agar (SDA), with chloramphenicol and gentamicin, with Cycloheximide. These tubes were incubated at $25^{\circ} \mathrm{C} .^{3}$

The cultures were observed twice weekly and discarded if there was no growth at the end of four weeks.

\section{Definitions}

1) Dermatophytic onychomycosis:

Cases in which dermatophytic culture was positive irrespective of positive or negative direct microscopy.

2) Yeast onychomycosis:

Cases in which nail specimen was positive in direct microscopy for yeasts irrespective of a positive or negative culture for yeasts.

3) Non dermatophytic onychomycosis:

Cases in which direct microscopy was positive for fungal hyphae at least once with growth of same nondermatophytic mould in culture at least twice from the same nail with no growth of dermatophyte on any occasion. ${ }^{4}$

4) Fungus positive group (Onychomycosis):

This includes all above these categories and the cases in which only direct microscopy was positive for fungal hyphae.

5) Fungus negative group (control group):

All the other patients with abnormal nails in the study group which did not fall in to fungus positive group were considered as the fungus negative (control) group.

Clinical classification of onychomycosis was followed as per Baran et al that included. ${ }^{5}$

1. Distal lateral subungualonychomycosis(DLSO)

2. Proximal onychomycosis (PO)

3. Superficial onychomycosis (SO)

4. Endonyxonychomycosis (EO)

5. Mixed pattern onychomycosis (MO)

6. Totally dystrophic onychomycosis (TDO)

7. Secondary onychomycosis 


\section{RESULTS}

Out of total 204 patients clinically suspected of having onychomycosis, $78(38.2 \%)$ were positive either for microscopy and/or culture (fungus positive group) and considered as cases of onychomycosis. Remaining $126(61.8 \%)$ patients were considered negative for onychomycosis (fungus negative group). Of the 78 patients $72(92.3 \%)$ patients were positive by direct microcopy and culture positivity was $57.7 \%$ (45/78) (Figure 1).

The commonest age group involved in patients with onychomycosis was young adults of $20-40$ years of age $46.1 \%(36 / 78)$ patients (Table 1$)$. In fungus positive group there was male preponderance, the male to female ratio being 2.2:1, though the difference was not statistically significant when compared with the negative group where ratio was $1.5: 1$. The age group of $<20$ years was statistically significantly associated with onychomycosis $(\mathrm{p}<0.05)$ $(15.4 \%)$ when compared with same age group in fungus negative patients $(6.3 \%)$.

In onychomycosis $52 / 78$ patients $(67.7 \%)$ had only fingernail involvement, $20 / 78$ (25.6\%) of patients had only toe nail involvement and $6 / 78(7.7 \%)$ had involvement of both toe nails and finger nails. When compared the type and material of footwear used in two groups, there was no significant difference in patients with toenail onychomycosis. Whereas usage of socks was significantly associated with onychomycosis (Table 2). It was noted that sedentary work was significantly associated with onychomycosis $(p<0.05)$ when compared with chemical related work and household work in housewives in onychomycosis group (Table 3).

There was no significant difference in fungus positive and fungus negative groups pertaining to hygienic practices like, daily bath, type of soap used, swimming and cutting of nails also history of contact with animals, sharing of beds, soaps, nail-cutter and common washing of clothes were comparable in both groups. Pertaining to involvement of nails in other family members, number was higher in fungus positive group (4\%) as compared to fungus negative group $(1.6 \%)$ but the difference was not statistically significant. Use of tobacco was noted in

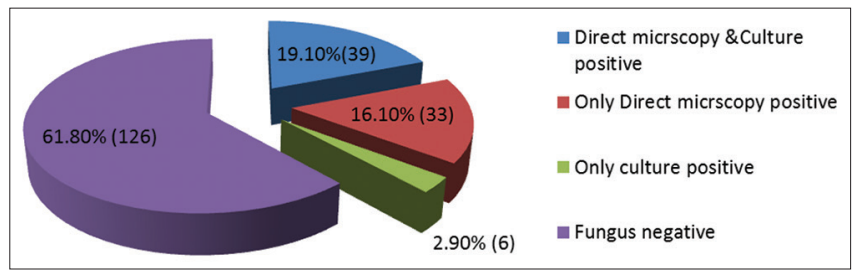

Figure 1: Direct examination and culture positivity in specimens clinically suspected of onychomycosis
$23 \%(18 / 78)$ patients which was significantly higher in fungus positive group when compared with the negative group in $17 / 126$ (13.5\%).(Table 4). While other addiction as alcohol/substance abuse had no significant difference between two groups.

\begin{tabular}{|c|c|c|c|c|}
\hline \multirow[t]{2}{*}{ Age in years } & \multicolumn{2}{|c|}{ Fungus positive (n) } & \multicolumn{2}{|c|}{ Total } \\
\hline & Male & Female & $\mathbf{n}$ & $\%$ \\
\hline$<20$ & 4 & 8 & 12 & $15.4^{*}$ \\
\hline $20-40$ & 28 & 8 & 36 & 46.1 \\
\hline $40-60$ & 14 & 7 & 21 & 27.0 \\
\hline$>60$ & 7 & 2 & 09 & 11.5 \\
\hline Total & 53 & 25 & 78 & 100 \\
\hline \multirow[t]{2}{*}{ Age in years } & \multicolumn{2}{|c|}{ Fungus negative (n) } & \multicolumn{2}{|c|}{ Total } \\
\hline & Male & Female & $\mathbf{n}$ & $\%$ \\
\hline$<20$ & 2 & 6 & 8 & $6.3^{*}$ \\
\hline $20-40$ & 34 & 21 & 55 & 43.7 \\
\hline $40-60$ & 27 & 22 & 49 & 38.9 \\
\hline$>60$ & 12 & 2 & 14 & 11.1 \\
\hline Total & 75 & 51 & 126 & 100 \\
\hline
\end{tabular}

\begin{tabular}{|c|c|c|c|c|c|c|}
\hline \multirow{2}{*}{$\begin{array}{l}\text { Socks } \\
\text { usage }\end{array}$} & \multicolumn{2}{|c|}{ Fungus positive } & \multicolumn{2}{|c|}{ Fungus negative } & \multicolumn{2}{|c|}{ Total } \\
\hline & $\mathbf{N}$ & $\%$ & $n$ & $\%$ & $n$ & $\%$ \\
\hline Yes & 14 & $53.8^{*}$ & 12 & $25.5^{*}$ & 26 & 35.6 \\
\hline No & 12 & 46.2 & 35 & 74.5 & 47 & 64.4 \\
\hline Total & 26 & 100 & 47 & 100 & 73 & 100 \\
\hline
\end{tabular}

\begin{tabular}{|c|c|c|c|c|}
\hline \multirow[t]{2}{*}{ Occupation } & \multicolumn{2}{|c|}{ Fungus positive } & \multicolumn{2}{|c|}{ Fungus negative } \\
\hline & $\mathbf{N}$ & $\%$ & $n$ & $\%$ \\
\hline Sedentary & 29 & 41.1 & 30 & 23.8 \\
\hline Housewife & 12 & 15.4 & 35 & 27.8 \\
\hline Chemical & 5 & 6.4 & 10 & 7.9 \\
\hline Other & 32 & 41.0 & 51 & 40.7 \\
\hline Total & 26 & 100.0 & 47 & 100.0 \\
\hline
\end{tabular}

\begin{tabular}{|c|c|c|c|c|c|c|c|}
\hline \multirow[t]{2}{*}{ Addictions } & \multicolumn{2}{|c|}{ Fungus positive } & \multicolumn{3}{|c|}{ Fungus negative } & \multicolumn{2}{|c|}{ Total } \\
\hline & $n=78$ & $\%$ & $n=126$ & & $\%$ & $n=204$ & $\%$ \\
\hline $\begin{array}{l}\text { Tobacco } \\
\text { chewing }\end{array}$ & 18 & $23.0^{*}$ & 17 & 13 & $0.5^{*}$ & 35 & 17.2 \\
\hline Smoking & 6 & 7.7 & 12 & 9 & 0.5 & 18 & 8.8 \\
\hline Alcohol & 3 & 3.8 & 3 & 3 & 0.8 & 6 & 2.9 \\
\hline Drugs & 0 & 0.0 & 0 & 0 & 0.0 & 0 & 0.0 \\
\hline Nil & 54 & 69.2 & 96 & 76 & 0.2 & 150 & 73.5 \\
\hline
\end{tabular}


Three patients in the onychomycosis group were HIV positive as compared to one in the negative group. In these 3 patients, one grew T. rubrum in culture and other two were only direct microscopy positive. These patients presented with distal lateral subungualonychomycosis. Other co-morbidities in patients with onychomycosis were skin atopy (1), sarcoidosis (1), Pulmonary TB (1) and hemiparesis (1). None of the diseases were significantly associated with onychomycosis.

Repeated nail trauma was the most common predisposing factor in both the groups. Cases with other predisposing factors like thumb sucking, nail biting, broad spectrum antibiotic and corticosteroid usage were too less in number to compare (Figure 2).

Fingernail involvement was more common in male \&female than toenail involvement in both groups of onychomycosis and non-fungal nail affection groups. The difference between fingernail \& toenail involvement in male and female was not significant in both groups. Multiple nails were involved in 52/78 (66.7\%) in fungus positive group and 85/126 (65\%) in the fungus negative group. These values are also comparable (Figure 3).
A total number of 72 out of 78 patients were positive for fungal elements by direct microscopy. By $\mathrm{KOH}$ mount, fungal elements could be detected in a total of 71 patients $(98.6 \%)$ and in $68(94.4 \%)$ of the patients by DMSO. Of these, 67 (93\%) showed concordant results (Table 5).

In the wet mounts, rapid clarification of background cellular debris was observed in DMSO preparations when observed after 10 minutes and one hour which was better than that achieved with $\mathrm{KOH}$ (Figure $4 \mathrm{a}$ and $\mathrm{b}$ ). However when observed after 24 hours, fungal hyphae in DMSO preparations appeared fragmented whereas in $\mathrm{KOH}$ preparations, the hyphae were intact (Figure $4 \mathrm{c}$,d). Yeasts in the nail were better appreciated in $\mathrm{KOH}$ than DMSO (Figure $4 \mathrm{e}, \mathrm{f}$ ).

Yeast onychomycosis constituted $30.7 \%$ of all the cases of onychomycosis. Other clinical types were seen in $69.3 \%$ of the patients. Distal lateral subungual onychomycosis and total destructive onychomycosis were the next commonly seen onychomycosis (Figure 5).

A total of 45 out of 78 patients with onychomycosis were positive by culture. $T$ rubrum was the most common

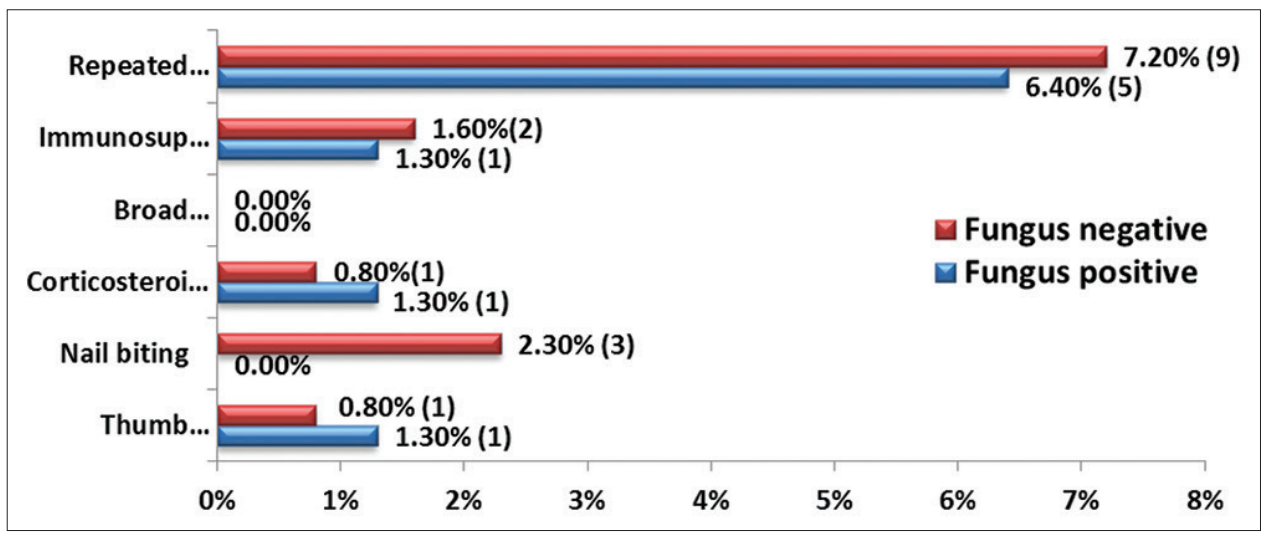

Figure 2: Predisposing factors in the fungus positive and fungus negative patients

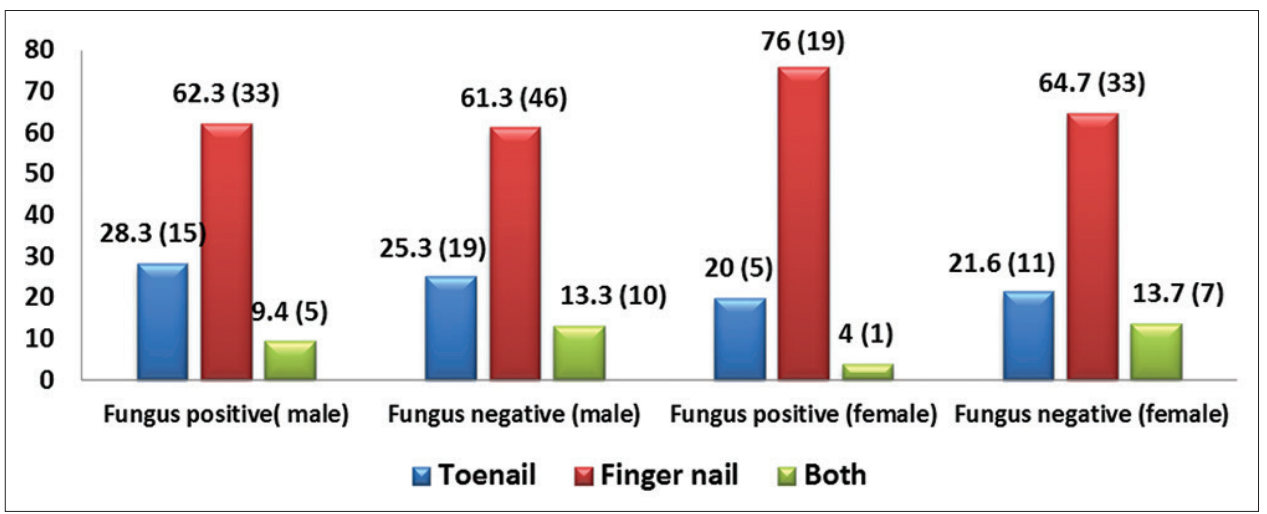

Figure 3: Site of nail involvement in males and females in the fungus positive and fungus negative patients 


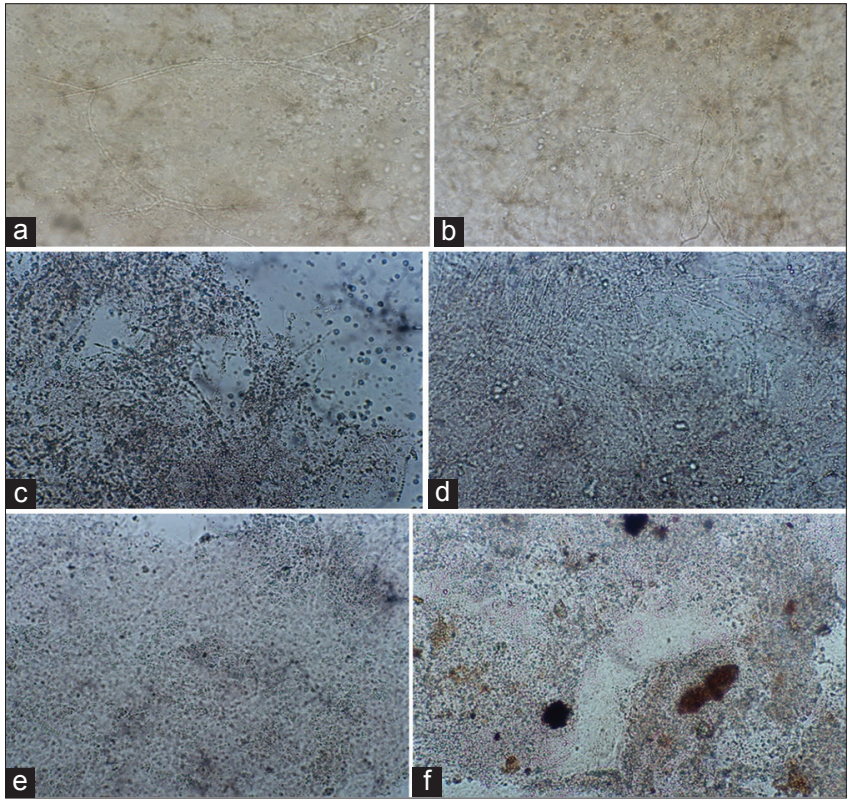

Figure 4: (a) Dimethylesulfoxide (DMSO), (b) potassium hydroxide $(\mathrm{KOH})$, Preparation of nail scrapings observed at 10 minutes (X200).(c) dimethylesulfoxide (DMSO), (d) potassium hydroxide $(\mathrm{KOH})$, Preparation of nail scrapings observed at 24 hour (X200). (e) dimethylesulfoxide (DMSO), (f) Potassium hydroxide (KOH) Preparation of nail scrapings showing large clumps of yeasts (X200) dermatophyte isolated. Micrsporum nanum was an unusual dermatophyte encountered. In yeast onychomycosis, C. parapsilosis was most frequently isolated. Aspergillus species were the commonest non-dermatophytes (Table 6).

Aspergillus ruberbrunneus was an unusual among nondermatophytes isolated (Figure 6).

The commonest change seen was ridging of the nail plate, followed by yellow discoloration of the nail $(44.9 \%)$ and other changes. Paronychia was observed in only $4 / 78(5.1 \%)$ of which three were having candidial onychomycosis.

All the etiologic varieties of OM were seen in fingernails as well as in toenails (Table 7). However Candida species were more commonly seen in fingernails than toenails.

Conventional treatment was given to patients in our study. A total of $11.5 \%$ (9/78) patient responded. Three patients did not respond to this treatment, 2 of them were having non-dermatopytic type of onychomycosis and one had dermatophytic onychomycosis. Unfortunately, large number of patients was either defaulter who did not

$\begin{aligned} & \text { Table 5: Comparison between potassium hydroxide (KoH) and dimethyl sulfoxide (DMSO) examinations } \\
& \text { in the fungus positive (onychomycosis) group }\end{aligned}$
\begin{tabular}{lccc} 
& $\mathrm{n}$ & & $\begin{array}{c}\text { Positive in both KOH } \\
\text { and DMSO preparation N }\end{array}$ \\
\hline $\begin{array}{l}\text { Total No. positive } \\
\text { in microscopy }\end{array}$ & Only KOH positive & $\begin{array}{c}\text { Only DMSO preparation } \\
\text { positive }\end{array}$ & 67 \\
\hline 72 & 4 & 1 & 67 \\
\hline
\end{tabular}

\section{Table 6: Etiological classification of onychomycosis with fungal isolates}

\begin{tabular}{|c|c|c|c|}
\hline $\begin{array}{l}\text { Etiological type of onychomycosis } \\
n=45(\%)\end{array}$ & Fungal isolates & Numbers & $\%$ \\
\hline \multirow[t]{6}{*}{ Dermatophytic onychomycosis $n=19$ (42.2) } & T.rubrum & 14 & 31.1 \\
\hline & T.mentagrophytes & 2 & 4.4 \\
\hline & Microsporum nanum & 1 & 2.2 \\
\hline & Microsporum audouinii & 1 & 2.2 \\
\hline & M.gypseum & 1 & 2.2 \\
\hline & Total & 19 & \\
\hline \multirow[t]{4}{*}{ Candidial onychomycosis n=14 (31.1) } & C.parapsilosis & 8 & 17.8 \\
\hline & C.albicans & 3 & 6.7 \\
\hline & C.tropicalis & 3 & 6.7 \\
\hline & Total & 14 & \\
\hline \multirow{9}{*}{$\begin{array}{l}0.2 \text { Nondermatophytic mould } \\
\text { onychomycosis } n=12(26.7)\end{array}$} & Aspergillus niger & 4 & 8.9 \\
\hline & Aspergillus candidus & 1 & 2.2 \\
\hline & Aspergillus ruberbrunneus & 1 & 2.2 \\
\hline & Fusarium solani & 2 & 4.4 \\
\hline & Aspergillus flavus & 1 & 2.2 \\
\hline & Chetomium globosum & 1 & 2.2 \\
\hline & Dematiaceous mould & 1 & 2.2 \\
\hline & Penicillium spp. & 1 & 2.2 \\
\hline & Total & 12 & \\
\hline Total & & 45 & 100 \\
\hline
\end{tabular}




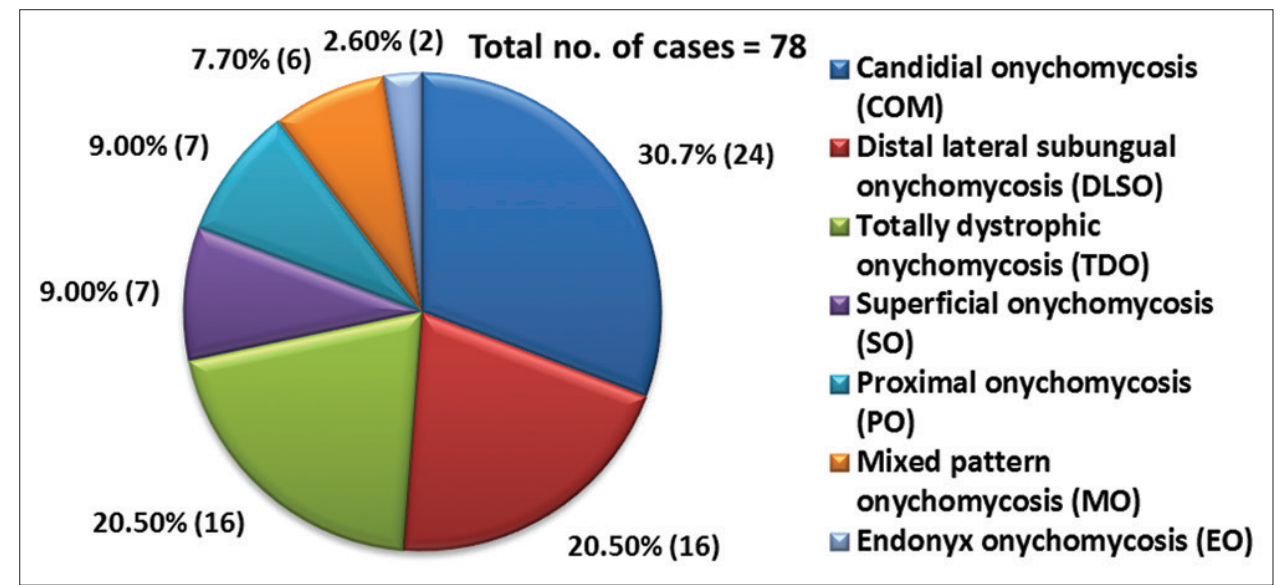

Figure 5: Distribution of clinical types of onychomycosis
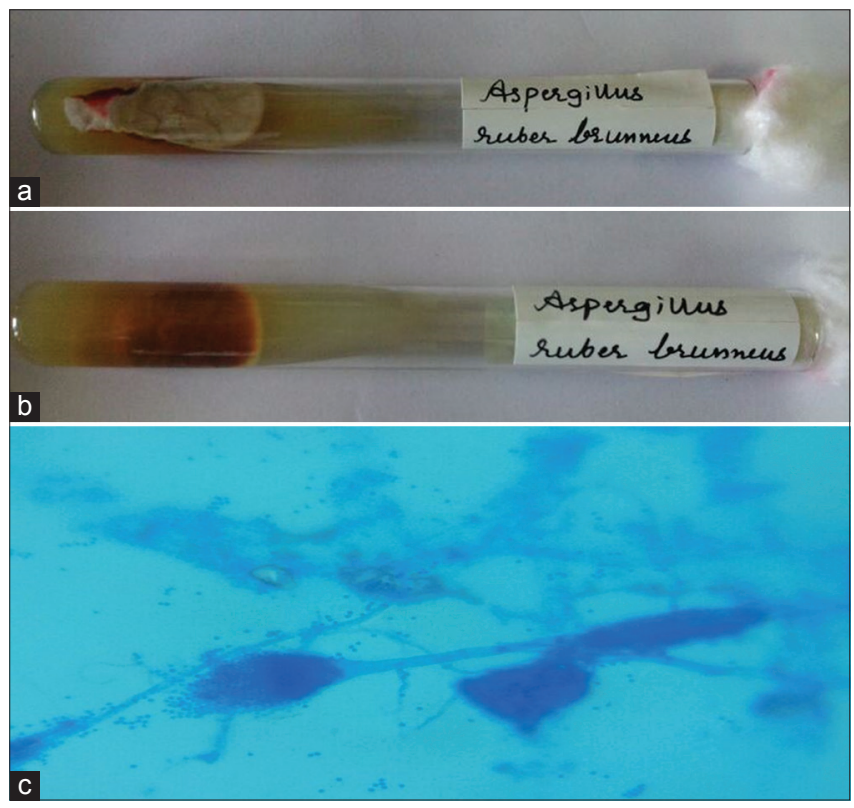

Figure 6: Aspergillus ruberbrunneus (a) Obverse, (b) Reverse, (c) Lactophenol cotton blue mount of slide culture (X200)

complete the treatment (38.5\%) or never followed up after initiation of treatment $(46.2 \%)$.

\section{DISCUSSION}

Onychomycosis is a chronic nail disease which if left undiagnosed and untreated leads to unsightly appearance and sometimes complications. In our study of 204 clinically suspected cases of onychomycosis, $38.2 \%$ were diagnosed of onychomycosis (Figure 1). Thus as many as $61.8 \%$ were wrongly clinically suspected, showing that relying only on pattern of nail changes is often misleading. Kaur et al have reported a $54.5 \%$ positivity for onychomycsois in her study of abnormal nails. ${ }^{1}$ Other studies have reported a $50.6 \%$ and $43.7 \%$ positivity. ${ }^{6,7}$ These figures are slightly higher than our report of $38.2 \%$. This may be because of difference in definition of cases to be considered as positive in both studies.

Kaur et al have reported prevalence of $7.8 \%$ in age $<15$ yrs. ${ }^{1}$ In our study a prevalence of $15.4 \%$ were seen in age upto 20 years and in age group upto 40 years, it was about $38.5 \%$ as compared to $16.5 \%$ figure by Kaur et al. Thus more than double prevalence is seen with advanced age. A higher prevalence in oldest age group of $>55$ yrs was observed by some workers. ${ }^{8}$ Faulty biomechanics in this age group due to bony abnormalities could be resulting in physical trauma and eventually onychomycosis. The highest prevalence was seen in our study in the age group of 20-40 years which was comparable to the figures in study by Veer $e t a \ell$ and Gupta et al..$^{10}$ In peadiatric age group mycotic infections of nails are considered to be uncommon. In our study, 15.4\% patients with onychomycosis belonged to peadiatric age group as compared to $7.8 \%$ as noted by Kauret al. ${ }^{1}$ Overcrowded environment and malnutrition could be subject to higher number of paediatric patients in our study.Interestingly, when this was compared to fungus negative group, the age group $<20$ years was found to be significantly associated with onychomycosis (Table 1).

The male predominance in onychomycosis has been noted in many studies. The male to female ratio of 3:1, 2.1:1 and 1.8:1 has been reported by various workers. ${ }^{9}, 10,11$ In our study it was 2.2:1. A male preponderance was also observed in the negative group (Table 1). Some investigators have suggested that estrogen exerts protective effect against onychomycosis as it has been seen frequently amongst postmenopausal female. ${ }^{12}$ On the other hand, testosterone might aggravate the condition since onychomycosis is seen more frequently in boys $>14$ yrs of age. ${ }^{13}$

Wet food, flour, fish, soap and chemicals have been reported to be associated with onychomycosis. ${ }^{2}$ In another 


\begin{tabular}{|c|c|c|c|c|}
\hline \multirow[t]{2}{*}{ Site affected } & \multicolumn{4}{|c|}{$n(\%)$} \\
\hline & Fingernail & Only toenail & Both & Total \\
\hline \multicolumn{5}{|l|}{ Culture positive $(n=45)$} \\
\hline Dermatophytic OM & $12(63.2)$ & $7(36.8)$ & 0 & $19(100)$ \\
\hline NDM OM & $6(50)$ & $4(33.3)$ & $2(16.7)$ & $12(100)$ \\
\hline Candidial OM & $11(78.6)$ & $2(14.3)$ & $1(7.1)$ & $14(100)$ \\
\hline \multicolumn{5}{|c|}{ Direct microscopy positive, culture negative $(n=33)$} \\
\hline Yeast & $6(60)$ & $3(30)$ & $1(10)$ & $10(100)$ \\
\hline Hyphae & $17(73.9)$ & $4(17.4)$ & $2(8.7)$ & $23(100)$ \\
\hline Total & $52(66.7)$ & $20(25.6)$ & $6(7.7)$ & $78(100)$ \\
\hline
\end{tabular}

study, white collar workers appeared to be at higher risk of infection when compared to other workers. ${ }^{11}$ In our study, $41.1 \%$ of patients were involved in sedentary work as compared to $23.85 \%$ in negative group which was significantly higher than that in housewives (15.4\%) and those associated with chemicals $(6.4 \%$ ) (Table 3$)$. It is possible that in sedentary group, more use of occlusive footwear, socks and trauma to the fingernails due to fine manual work could be the contributing factors. There were more cases in fungus negative group than in positive group in cases with history ofcontact with other materials like wood, water, soap, disinfectants and food. Other factors like daily bath, type of soap, etc were not associated with onychomycosis in our study when compared with fungus negative group. Family history of onychomycosis as potential source of fungal infection has been reported in some studies. ${ }^{14,15}$ but there was no significant difference in family history of fungus positive and negative groups.

Artificial material andocclusive footwear may promote sweating and trauma to the nail resulting in toenail onychomycosis. In our study, there was no significant association with type or material of footwear. However a significant association with onychomycosis was seen in those wearing socks in our study (Table 2). Use of nylon socks has been found to be more frequent in farmers leading to onychomycosis in a study from Turkey. ${ }^{16}$ Extreme manicure, sharp pointed shoes, nail biting and finger sucking may develop to OM. ${ }^{2}$

Onychomycosis is a common condition with multifactorial etiology including both genetic and acquired factors being responsible. Smokers have been found to have greater prevalence of onychomycosis. ${ }^{17}$ In our study however, the number of smokers was not significantly higher in fungus positive group. Tobacco addiction however was significantly associated with fungus positive group as compared to fungus negative group (Table 4). Tobacco usage is known to be associated with release of nicotine which has an effect on the peripheral blood supply. ${ }^{18}$ Peripheral vascular disease was also found to be a contributory factor.
Some studies have not found diabetes as co-morbidity in OM. ${ }^{11,19}$ In our study also it was not found to be significantly associated with $\mathrm{OM}$ when compared to the fungus negative group. On other hand, Onychomycosis has been found to be more common in diabetic patients than in non-diabetics by another study. ${ }^{20}$

Toenails have been found to have lesser blood supply than fingernail. Toenail was found to be more commonly involved than fingernail in $56.9 \%$ of patients as compared to $32.3 \%$ of patients in a study from Shimla. ${ }^{10}$ This appears to be finding in studies from temperate climate, possibly of wearing closed footwear for long hours and walking for distances in hilly areas. In our study, however, fingernails were found to be more commonly involved than toenails which could be due to warm climate in the region and subsequently less use of occlusive footwear. Veer $e t$ al also report more common involvement of fingernails than toenails in $63 \%$ and $21 \%$ respectively. ${ }^{9}$ In their study fingernail and toenail infection ratio was 3:1. In our study it was 2.2: 1 (Table 3).

Gupta et al have reported involvement of 10-15 nails in $4.6 \%$ of patients, $5-10$ in $36.1 \%$ of patients and $<5$ nails in $5.36 \%$ of patients $(59) .{ }^{10}$ In our study multiple nail involvement was seen in $66.6 \%$ and single nail involvement in $33.3 \%$. It was not significantly different than fungus negative group. Similarly involvement of $1-2$ nails in $31.2 \%$ of the patients was observed in another study ${ }^{6}$

Direct examination with $\mathrm{KOH}$ offers rapid diagnosis of $\mathrm{OM}$ and also valuable information on type of etiological agents involved that can be co-related with culture findings. But the microscopic recognition of fungus in nail requires considerable experience and practice. A study by Singh et al compared 40\% DMSO and 10\% $\mathrm{KOH}$ for observing skin, hair and nail samples.For nail clipping,they found rapid clearing within 10 minutes with DMSO and 30 minutes with $\mathrm{KOH}$ were required. ${ }^{23}$ In our study, $\mathrm{KOH}$ was found to be more sensitive in detecting fungi. As far as clarity concerned, in DMSO background was cleared in 10 minutes which was not achieved with 
$\mathrm{KOH}$ (Figure 4a and b). When observed after 24 hours, the fungal elements in DMSO developed fragmentation and artifacts but in $\mathrm{KOH}$ they were still well seen (Figure $4 \mathrm{c}$ and d). Thus it appears that DMSO clears the background more effectively than $\mathrm{KOH}$ however $\mathrm{KOH}$ retains fungal morphology better. In essence, $\mathrm{KOH}$ found to be better option for observing fungi in our study. Fungi could be seen effectively in both the preparations.

In a study by Veer et al, 34 patients were found to be culture negative amongst the 72 , who were direct microscopy positive. ${ }^{9}$ On the other hand, 16 were negative in direct microscopy out of which 5 were culture positive. Their findings are in conformity with our findings (Figure 1). The reason for culture negativity in spite of microscopic evidence could be deep location of fungi in tissue, nonviability of fungi or non-representative sample being inoculated on culture media. In our study, microscopy negative and culture positive cases were 6 . This could suggest low fungal load. As isolation of dermatophyte is essentially to be considered as significant from nail sample, despite microscopy being negative, these were considered as pathogen in OM. Similar was not true with nondermatophytes and yeasts since they may be contaminants in nails. Hence such isolates if microscopically negative were disregarded in our study. Kaur et al have reported direct microscopy positive $136(34 \%)$ of specimens out of 400 and out of these 38 were positive in culture. There appears to be $28 \%$ negativity in despite direct microscopy positivity. ${ }^{1}$

Amongst the different clinical types of OM, distal lateral subungual $\mathrm{OM}$ is supposed to be the commonest clinical type. ${ }^{5}$ Candidial onychomycosis has been reported in $6.6 \%$ patients in a study from India. ${ }^{1} \mathrm{~A}$ higher prevalence of $27.3 \%$ of cases has been reported in another study. ${ }^{19} \mathrm{In}$ our study yeast OM was high at $30.8 \%$ followed by DLSO (Distal Lateral Subungual Onychomycosis) and TDO (Total Destructive Onychomycosis) in $20.5 \%$ each. In our study we have also included cases which were also positive for yeasts in direct microscopy despite of them being negative in culture. This factor which has not been considered in other studies could be the reason for higher number of cases reported in our study.

In a study from Lahore, DLSO was found in $47 \%$ followed by candidial OM in $36 \% .{ }^{24}$ Other varieties noted were TDO $(12 \%), \operatorname{SOM}(2 \%)$ and PO $(2 \%)$. These figures are in conformity with our study. In the Indian study by Veer et al a $4.7 \%$ prevalence of superficial OM was observed. ${ }^{9}$ The other types described were DLSO (70.3\%), COM (14.6\%), TDO (7.7\%) and PSO (0.7\%). DLSO as commonest variety was seen in HIV patients. In our study also all 3 patients had DLSO.
OM caused by dermatophytes and yeasts are more common varieties seen in our study. Kaur et al have also reported dermatophytic $\mathrm{OM}$ as commonest variety but in their study, NDM are as high as $44.3 \% .^{1}$ In their study, out of 88 dermatophytes, 45 could not be speciated and in remaining, T. mentagrophytes, T. rubrum, $M$ gypsium, T. tonsurans, E floccosumwere seen. Among NDM, Aspergillus was the commonest (39/69), followed by Rhizopus in (14), mucor in (12). The other NDM were Fusarium, Alternaria, Penicillium, Bipolaris, Schedosporium and Culvularia. The authors have also reported mixed infection however a percentage of it was not provided. Dermatophytes were most common pathogen in another study from central India in $26.36 \%$ cases. In this T. rubrum was commonest isolate followed by T. Verrucosum and E.floccosum Candida albicans was isolated from $24.2 \%$ and NDM in 29 patients. ${ }^{25}$ T. rubrum as commonest etiological agents was noted also by Perea followed by T. mentagrophytesvarinterdigitale and T. tonsurans. ${ }^{11}$ Das et al also reports high prevalence of dermatophtic fungi in 22/44 number of cases. ${ }^{19} \mathrm{~T}$ rubrum as the commonest isolate followed by T. metagrophytes is like found in our study. Amongst yeasts, Candida albicans has been reported as commonest by these authors. Other isolates have not been speciated. Among NDM, A. niger was commonest isolate in this study followed by Fusarium. This finding is also similar to our study (Table 6). Thus it appears that a wide array of fungi can cause OM.

In India, T. rubrum and T. mentagrophytes are most commonly isolated agents from OM. Other dermatophytes are less commonly encountered as T. violaceum, ${ }^{7}$ T.tonsurans, T. verrucosum ${ }^{10}$ M. nanum and E.floccosum. ${ }^{6,7}$ Candidial OM is mostly caused by C. albicans. ${ }^{9}$ Other species reported are C Candia parapsilosis ${ }^{26}$, Candida glabrata ${ }^{22}$, Candida kruse ${ }^{22}$ and Candida guilliermondii. ${ }^{2}$ Many non-dermatophytes have been reported to cause OM. Scopuliaropsis brevicaulis, species of Aspergillus, Acremonium, Fusarium and dematitious moulds like Neoscytilidium dimideatum, Cladosporium, Alternaria, Cheatomium globosum have also been reported. ${ }^{2}$ In Indian reports the commonest non-dermatophyte mould reported is $A$.niger. ${ }^{21,9}$ In our study, M. nanum was the unusual isolate from one patient. She was a 50 years female working as staff nurse. M. nanum does not appear to be hitherto reported to be as a cause OM from India. Another unusual isolate, in our study was Aspergillus ruberbrunneus reported in one patient. It is a plant pathogen also reported to cause hay fever in farmers but not yet reported to causeonychomycosis. Our patient was a 50 years old retired fitter, had exposure to chemicals in his occupation. He had TDO and taken conventional treatment by dermatologist but did not respond and was lost to follow up.

DLSO can be caused by dermatophytes as well as non-dermatophytes as Aspergillus, so also like Fusarium. ${ }^{2}$ 
WSO is caused by dermatophytes, as T. metagrophytes and T. rubrumbut, NDM can also cause this infection. Proximal onychomycosis is primarily caused by Trichophyton rubrum. ${ }^{26}$ Fusarium has also been described in this type of OM. ${ }^{2}$ Trichophyton rubrum has been reported as the predominant pathogen in Distal Lateral Subungual Onychomycosis, Endonyx Onychomycosis, Superficial Onychomycosis, Proximal Onychomycosis and Total Destructive Onychomycosis followed by Trichophyton mentagrophytes in our study.

Several morphological changes in the nails may occur as a result of disease process. Ridging was the commonest morphological abnormality seen in our patients. The affected nails show number of changes like subungual hyperkeratosis, pits and lamellar splits, hyperkeratosis and discoloration which could be as a result of pigment accumulation or cellular alteration. In our study, pigmentation was found in $33.3 \%$ of patients. Das et al have reported black pigmentation as the commonest in his study. ${ }^{19}$ In our study, yellow pigmentation was most common and was seen in $44.9 \%$ of patients and it was seen in all three etiological types of OM. Pigmentation of nails could result from pigment produced by matrix melanocytes. $\mathrm{OM}$ is generally a chronic condition of nail for which few patients seek medical attention unless they have symptoms like paronychia. In our patients, paronychia was observed in only $5.1 \%$ of the patients.

All etiological categories of $\mathrm{OM}$ were found to be more common in fingernail than toenails like dermatophytic, non-dermatophytic and candidial onychomycosis and also only microscopically proven onychomycosis. Candidial onychomycosis was found to be significantly higher in fingernails than toenails in our study. Szepietowski J C et al have found dermatophytic infection was commonest in toenails and fingernail followed by candidial infection and the least common was moulds. ${ }^{7}$ But it was seen that in fingernails the rate of yeast infection was much higher in $32.8 \%$ than in the toenails $(9.8 \%)$. Even among our cases the rate of yeast infection in fingernails was much higher than the toenails (Table 7).

Clinical cure rates for OM have often been reported to be less than $15 \%{ }^{12}$ Until an optimal treatment becomes available, however, onychomycosis will remain to be one of the difficult diseases to treat. Its epidemiology and ecology is complex and less understood. Optimal treatment is far less from achieved. Conditions are expected to be worse in India where attention to personal health takes a backseat with other pressing issues. There is need for public awareness and education as if left untreated onychomycosis tends to destroy the nail plate. Direct microscopy and culture are the diagnostic gold standards. ${ }^{13}$ In India and other tropical countries there is scanty data available even at this point of time. There is need to strengthen the patient education and counseling for follow up which is often very poor as was found in our study.

\section{REFERENCES}

1. Kaur R, Kashyap B and Bhalla PA. Five year survey of onychomycosis in New Delhi, India: epidemiological and laboratory aspects. Indian J Dermatol 2007;52: 39-42.

2. Torres-Rodriguez JM and Lopez-Jodra O. Epidemiologyofnail infection due tokeratinophilic fungi. Rev Iberoam Micol 2000; 17:122-135.

3. Koneman EW and Roberts GD. Practical Laboratory Mycology. $3^{\text {rd }}$ ed. Baltimore; Williams and Wilkins, 1985: 21-106.

4. Gupta AK, Cooper EA, Mac Donald P and Summerbell RC. Utility of inoculum counting (Walshe and English criteria) in clinical diagnosis of onychomycosis caused by nondermatophytic filamentous fungi. J Clin Microbiol 2001;39:2115-2121.

5. Baran R and Hay RJ. Onychomycosis: A proposed revision ofthe clinical classification. J Am Acad Dermatol 2011;19: 21.

6. Yenişehirli G, Bulut $Y$, Sezer E and GündayE. Onychomycosis infections in the Middle blacksea region, Turkey. Int J Dermatol 2009; 48:956-959.

7. Szepietowski JC. Selected clinical aspects of onychomycosis. Mikollek 2004; 11: 119-128.

8. Scher R and Bran R. Onychomycosis in clinical practice: factors contributing to recurrence. Br J Dermatol 2003;149: 5-9.

9. Veer P, Patwardhan NS and Damle AS. Study of onychomycosis: prevailing fungi and pattern of infection.Indian J Med Microbiol 2007; 25:53-56.

10. Gupta M, Sharma NL, Kanga AK, Mahajan VK and Tegta GR. Onychomycosis: clinico-mycological study of 130 patients from Himachal Pradesh, India. Indian J Dermatol Venereol Leprol 2007;73:389-392.

11. Perea S, Ramos MJ, Garau M, Gonzalez A, Noriega AR and Palacio A. Prevalence and risk factors of Tinea unguium and Tinea pedis in the general population in Spain. $\mathrm{J}$ Clin Microbiol 2000;38:3226-3230.

12. Daniel CR III. Tinea unguium. J Miss State Med Assoc 1986;27:295-296.

13. Elewski BE and Hay RJ. Update on the management of onychomycosis: highlights of the third annual international summit on cutaneous antifungal therapy. Clin Infect Dis 1996; 23:305-312.

14. Kim DM, Suh MK and Ha GY. Onychomycosis in children: An experience of 59 cases. Ann Dermatol 2013; 25(3): 327-334.

15. Romano C, Papini M, Ghilardi AG and Gianni C. Onychomycosis in children: a survey of 46 cases. Mycoses 2005;48: 430-437.

16. Sahinl, Kaya D, Parlak AH, Oksuz $S$ and Behcet $M$. Dermatophytoses in forestry workers and farmers. Mycoses 2005; 48:260-264.

17. Gupta AK,Gupta MA, Summerbell RC, Cooper EA, Konnikov N and Albreski D. The epidemiology of onychomycosis: possible role of smoking and peripheral arterial disease. J Eur Acad Dermatol Venereol 2000;14:466-469.

18. Tisdale JE, Patel RV, Webb CR, Borzak S and Zarowitz BJ, Prarrythmaic effects of intravenous vasopressors. Ann Pharmacother 1995: 29; 269-281.

19. Das KN, Das $S$, Ghosh $P$, Dutta RN, Sengupta SR and Bhattacharya S. A study on etiological agents and 
clinicomycological correlation of fingernail onychomycosis in Eastern India. Indian J Dermatol 2008:53(2); 75-79.

20. Winston JA and Miller JL. Treatment of Onychomycosis in diabetic patients. Clinical Diabetes 2006; 24(4):160-166.

21. Surjushe A, Kamath R, Oberai C, Saple D, Thakre M, Dharmshale $\mathrm{S}$, et al. A clinical and mycological study of onychomycosis in HIV infection. Indian J Dermatol Venereol Leprol 2007;73:397-401.

22. Jayatilake JAMS, Tilakaratne WM and Panagoda GJ Candidal onychomycosis: A Mini-Review.Mycopathologia 2009;168:165-173.

23. Singh $S$ and Beena PM. Comparative study of different microscopic techniques of isolation of dermatophytes. Indian $\mathrm{J}$ Med Microbiol 2003;21:21-24.

24. Bokhari MA, Hussain I, Jahangir M, Haroon TS, Aman $S$ and Khurshid K. Onychomycosis in Lahore, Pakistan. Int J Dermatol 1999; 38:591-595.

25. Das S, Goyal R and Bhattacharya SN. Laboratory based epidemiological study of superficial fungal infections. Indian $\mathrm{J}$ Dermatol 2007;34:248-253.

26. Figueiredo VT, Santos D, Resende MA and Hamdan JS. Identification and in- vitro antifungal susceptibility testing of 200 clinical isolates of Candida spp.responsible for fingernail infections.Mycopathologia 2007;16:27-33.

\section{Authors Contribution:}

AFI - Conceptualized study, literature search, data collection, statistically analyzed and interpreted, prepared first draft of manuscript and critical revision of the manuscript; TUM - Concept and design of the study, reviewed the literature, manuscript preparation and critical revision of the manuscript, BSM - Concept, review of literature and helped in data collection and critical revision of the manuscript; DR and PM - Concept of study, collected data and review of study.

Source of Support: Nil, Conflict of Interest: None declared. 